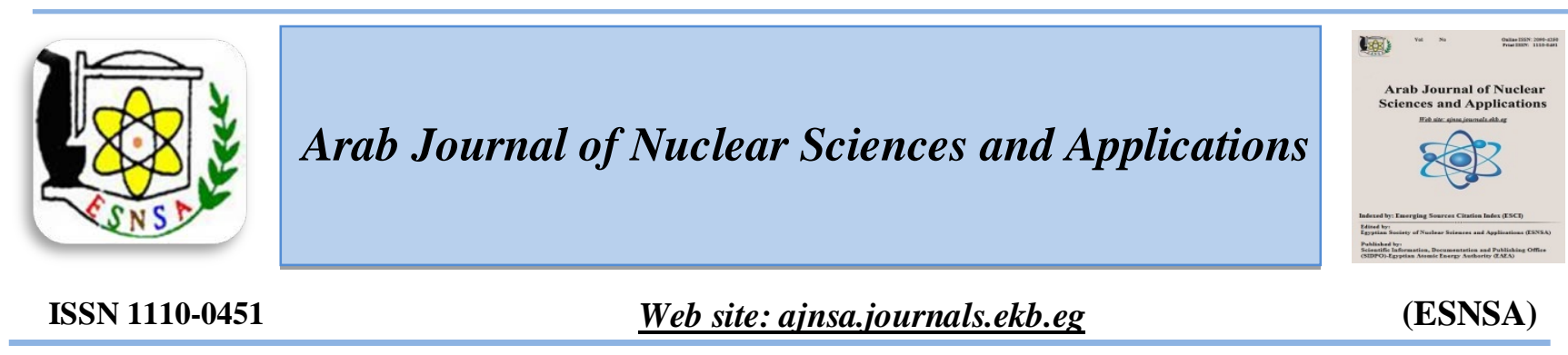

\title{
Spectroscopy Measurements of Laser-Induced Molybdenum Plasma
}

\author{
Dalia Abdrabou $^{\text {a }}$ M. A Khedr ${ }^{\mathrm{b}}$, N. Fathi ${ }^{\mathrm{b}}$, A M Hussein ${ }^{\mathrm{a}}$, Th M El Sherbini ${ }^{\mathrm{c}}$ \\ (a) Misr University for Science and Technology, 6 October, Egypt \\ (b) National Institute of Laser Enhanced Science, Cairo University, Giza, Egypt \\ (c) Laboratory of Laser and New Materials, Department of Physics, Faculty of Science, Cairo University, Giza, \\ Egypt
}

Received 21 ${ }^{\text {st }}$ Dec. 2017 Molybdenum plasma in air at atmospheric pressure was carried out by utilizing Nd-YAG laser at Accepted $23^{\text {rd }}$ April fundamental wavelength $1064 \mathrm{~nm}, 0.206 \mathrm{~mJ}$ input energy, $5 \mathrm{~ns}$ pulse duration and repetition rate $10 \mathrm{~Hz}$. After plasma formation, the optical fiber was used to collect plasma emission. The spectral analysis was displayed in the Local Thermal Equilibrium (LTE) state. Electron temperature was estimated by Boltzmann plot method and electron density was calculated from Stark broadening and compared with electron density of $\mathrm{Ha}$-line at $\mathbf{6 5 6 . 3} \mathrm{nm}$ which has reliable values. The temperature of plasma and density were evaluated for different positions along the expansion axis. The influence of the input power on plasma was studied. The electron density varied from $5 \times 10^{18}$ to $3.5 \times 10^{19} \mathrm{~cm}^{-3}$. These parameters are important in environmental and industrial application. At distance of $10 \mathrm{~mm}$ from the target, the electron temperature has a minimum value while the electron density shows a maximum value.

Keywords: Molybdenum; LIBS; Electron density; Electron temperature; Hydrogen Ha-line

\section{Introduction}

The investigation of molybdenum is used in various fields, such as space-exploration, and industrial applications. Commercial Molybdenum is an element used for the production of optical diagnostics metal mirrors as in ITER [1], this is due to the good properties of Molybdenum such as high melting point, low coefficient of thermal expansion and high level of thermal conductivity.

Optical Emission Spectroscopy has importance worldwide for describing materials based on laser induced plasma (LIP-technique) [1, 2]. It is used a spectroscopic analytical method [3, 4]. It is a technique used to release light from plasma generated through interaction of a high power lasers with matter (solid, liquid or gase). The light emission of plasma can give information about "spectral signatures" of many different materials in liquid solid or gas state [5] . Plasma diagnosis can be done through the measurement of electron density $\left(\mathrm{N}_{\mathrm{e}}\right)$ and temperature $\left(\mathrm{T}_{\mathrm{e}}\right)$ through optical emission spectroscopy (OES) [6]. The electron temperature and density were measured for different materials such as Sulphur [7], Silicon [8], Zinc [9, 10], Aluminum [11], Iron [12], Fe [13], Mo [14], Magnesium [6, 15], Copper [16] ....etc. In a continuous study in this important field, we monitor the plasma parameters of Mo solid sample via measuring electron temperature and electron density utilizing LIBS technique. In this article, we measured plasma parameter $\left(\mathrm{T}_{e}\right.$ and $\left.\mathrm{N}_{\mathrm{e}}\right)$ for Mo solid samples at different input powers and distances from the target.

\section{Experimental Setup}

In Fig (1), a Nd:YAG laser emitting pulses with $0.206 \mathrm{~mJ}$ energy and $5 \mathrm{~ns}$ pulse time duration was concentrated on a molybdenum solid sample with a laser fluence of $6 \mathrm{~J} / \mathrm{cm} 2$. The laser pulse passes

Corresponding author: dalia.saad@must.edu.eg

DOI: 10.21608/ajnsa.2018.2359.1027

(C) Scientific Information, Documentation and Publishing Office (SIDPO)-EAEA 
through a lens with a focal length of 10 to prevent any breakdown of the ambient air in front of the sample. The plasma was created by laser interaction with the sample. The emitted spectra from the sample were collected utilizing an optical fiber. A computer is used to record the emitted spectra from plasma plume using oplenic spectrophotometer which moves the holder to avoid cratering. The analysis of this emission is used to display information about the elemental structure, and the basic physical processes in plasma [8]. The spectrum is used to calculate the electron density $\left(\mathrm{N}_{\mathrm{e}}\right)$ and the electron temperature. The electron number density $\left(\mathrm{N}_{\mathrm{e}}\right)$ was measured from the Stark broadening parameter. The electron temperature is calculated from Boltzmann plot. Moreover the variations of electron temperature and electron density were studied as a function of input power and distance.

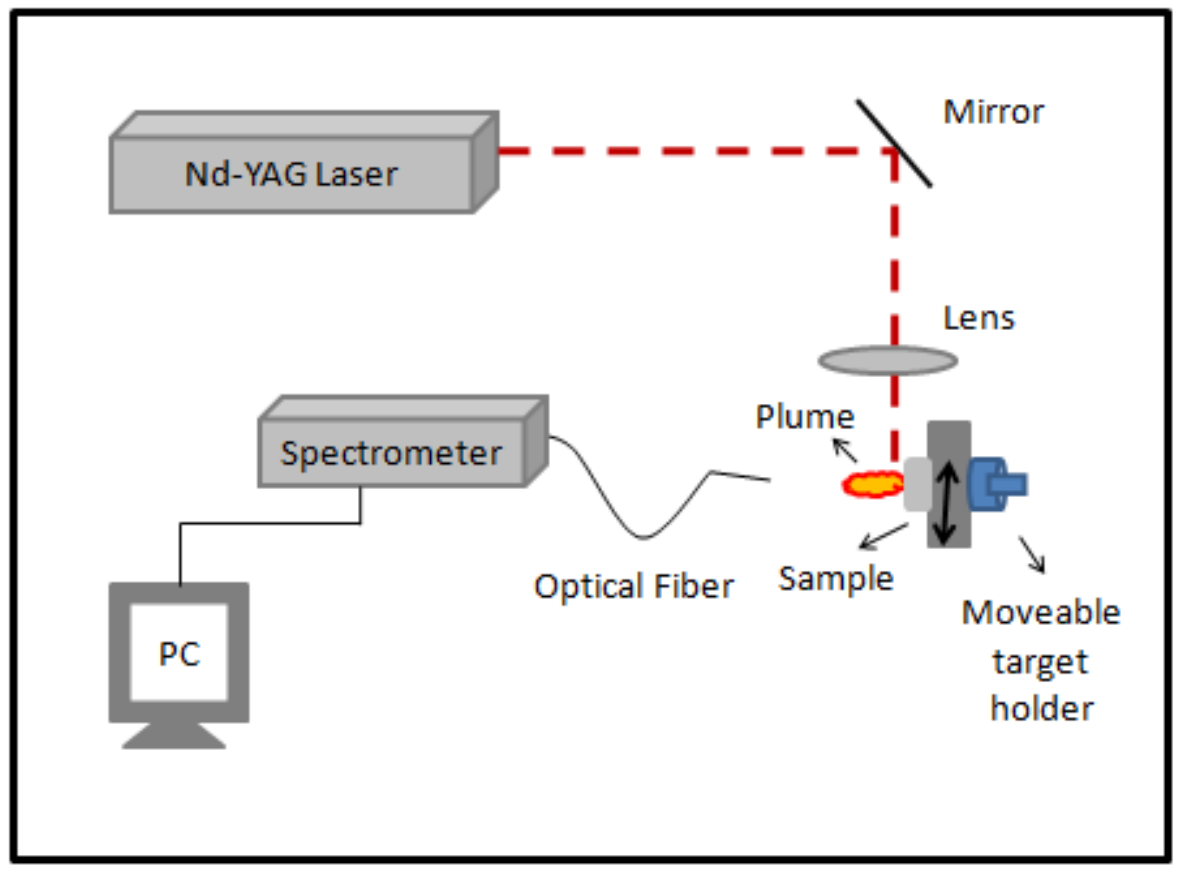

Fig. (1): the diagram of the experimental setup of LIBS

Plasma electron density is used to determine the thermodynamic plasma state. The electron densities have been determined from Stark broadened line profile of an isolated line of neutral atoms using eq (1). From the experimental results of electron impact width parameter of Mo lines was measured as $(0.01 \pm 0.02 \mathrm{~nm})$ [17]. The electron density can be determined using the Stark broadening of the spectral lines. This effect shows itself on the shape of Lorentzian broadening to the emitted line of full width at half maximum (FWHM) $\Delta \lambda_{0}$ for neutral atoms, the following expression can be used, after neglecting the ion contribution to the broadening, to measure the electron density[6, 18-20]:
Measurement of plasma electron density $\left(N_{e}\right)$

$$
\Delta \lambda_{1 / 2}=2 \omega\left(\frac{N_{e}}{10^{16}}\right)
$$

Where, $\Delta \lambda_{1 / 2}$ is the Full Width at Half Maximum (FWHM) of the spectral line, $\omega$ is the electron impact broadening parameter, and $\mathrm{N}_{\mathrm{e}}$ is the electron number density.

Measurement of electron density using the $\mathrm{H}_{\alpha}$-line For the hydrogen $\mathrm{H} \alpha$-line, the following expression is used to calculate the electron density [10], 
$n_{e}\left(H_{\alpha}\right)=8.02 \times 10^{12}\left(\frac{\Delta \lambda_{1 / 2}}{\alpha_{1 / 2}}\right)^{3 / 2}$

where, $\alpha_{1 / 2}$ is the half width of the reduced Stark profiles in $\AA$, accurate values of $\alpha_{1 / 2}$ for the Balmer series can be found in Ref. [1].

\section{Measurement of plasma temperature}

The electron temperature was measured from the relative intensities of the observed lines using the Boltzmann plot method. Three emission lines of Mo (I) is used to calculate the electron temperature under the condition of local thermodynamic equilibrium (LTE) [21].The following expression has been used to calculate the plasma temperature [7, 22]:

$\ln \left(\frac{I_{k i} \lambda_{k i}}{A_{k i} g_{k}}\right)=\ln \left(\frac{h c N_{o}}{4 \pi U_{o}}\right)-\frac{E_{k}}{K T_{e}}$

Where, $I_{k i}$ is the spectral intensity, $\lambda_{k i}$ is the wavelength, $A_{k i}$ is the transition probability and $g_{k}$ is the statistical weight of the upper state $(\mathrm{k})$ respectively. The subscript numbers indicate that different lines $N_{o}, U_{o}$ are the population density and the partition function of the atom at temperature Te. The constants h, c are the Planck constant and speed of light . $E_{k}$ is the energy of the upper level, $\mathrm{k}$ is the Boltzmann constant and $\mathrm{T}_{\mathrm{e}}$ is the electron temperature.

\section{Results and Discussion}

Fig. (2) Shows the emission spectrum in the range from $400-700 \mathrm{~nm}$ as recorded by the Oplenic spectrophotometer at three neutral lines of molybdenum (465.5, 498 and $517 \mathrm{~nm}$ ) as well as the $\mathrm{H} \alpha$-line at $656.27 \mathrm{~nm}$.

A plot of $\ln (\mathrm{I} / \mathrm{Ag})$ versus the term energyEk gives a straight line with a slope equal to $(-1 / \mathrm{KTe})$. Electron temperature along plasma expansion axis was determined from Boltzmann plot via eq (3).

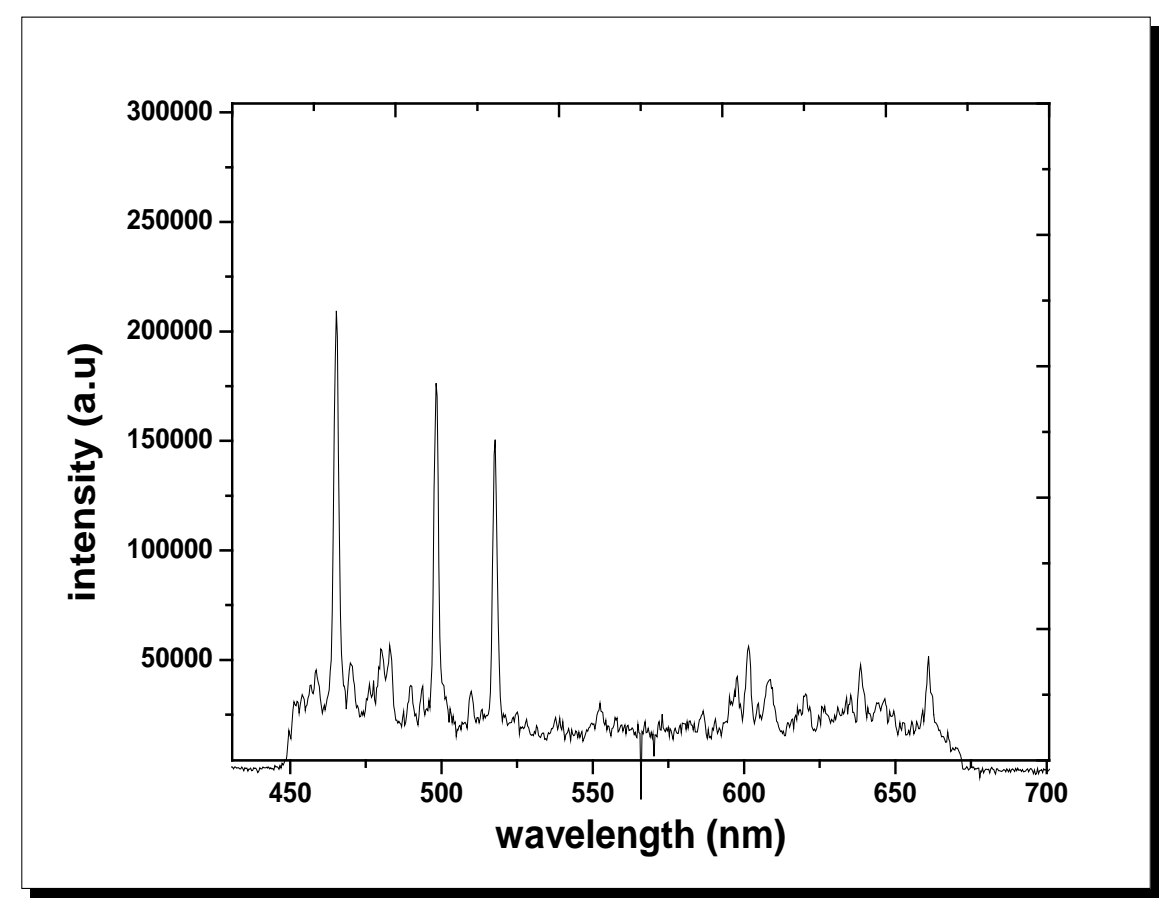

Fig. (2): the spectrum emitted from plasma using the fundamental $(1,064 \mathrm{~nm})$ wavelength of Nd-YAG laser at different input powers and positions 


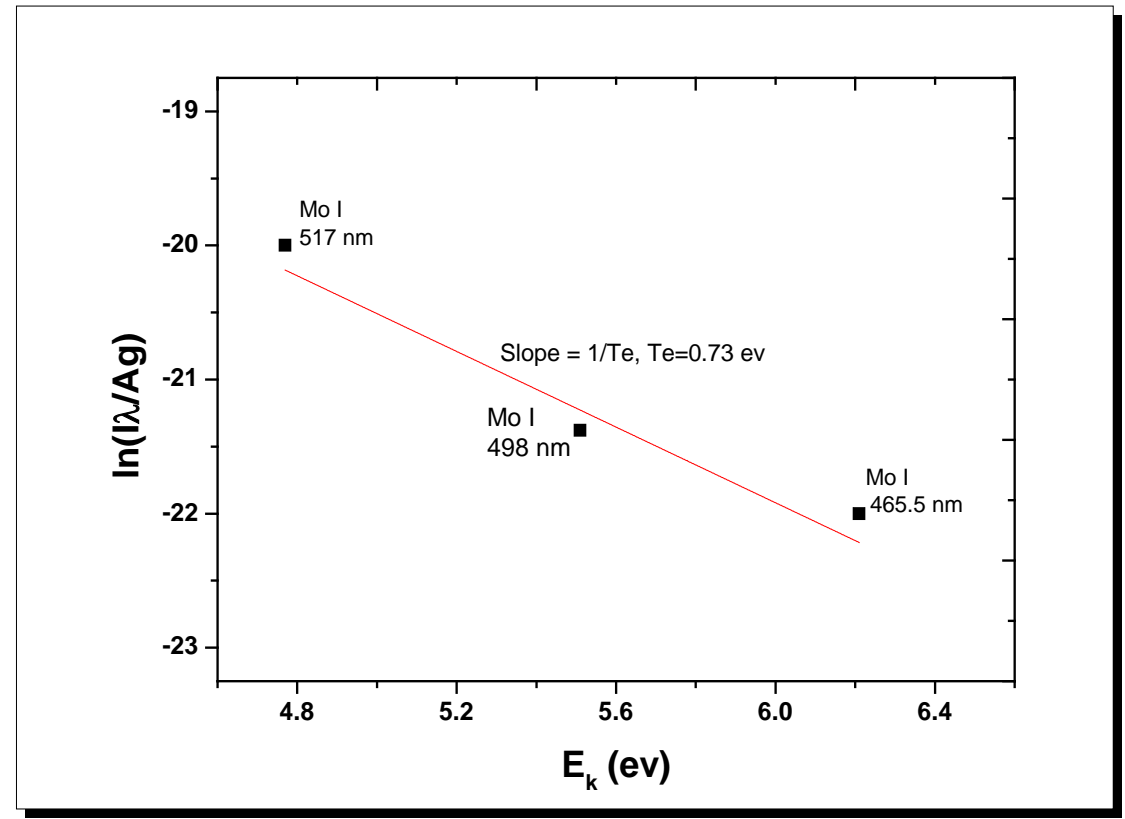

Fig. (3): Boltzmann plot utilizing the Mo I lines at fixed power $0.875 \mathrm{~W}$

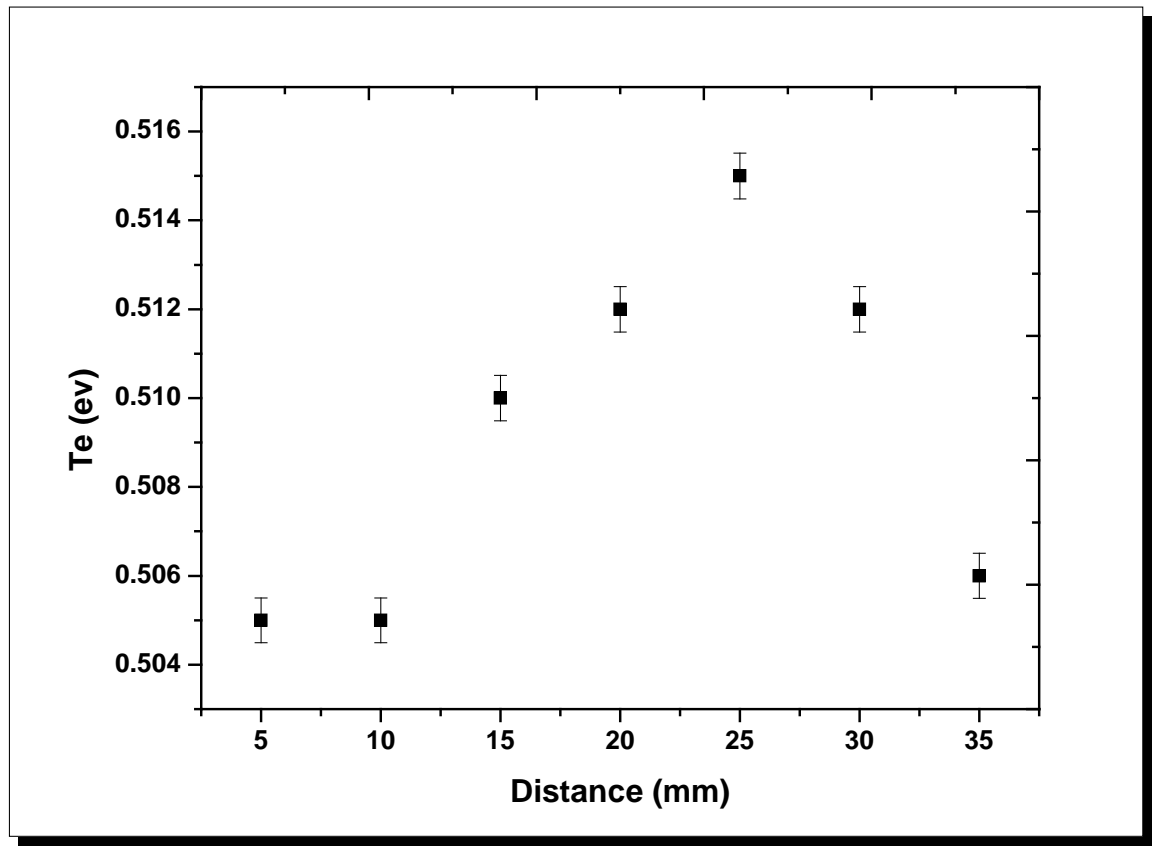

Fig. (4): Variation of the electron temperature with the distance using the fundamental $(1064 \mathrm{~nm})$ wavelength of the Nd-YAG laser at fixed power $0.875 \mathrm{~W}$

Fig (4) shows the variation in the electron temperature with the distance. The temperature has a maximum at the center of the plume and is minimum at the ends because of recombination and cooling of the plasma., the result is in agreement with the data reported in a previous study [23]. When the plasma expands, it thermalizes by transferring the energy to its surroundings. Moreover, the temperature decreases rapidly within a short distance from the sample because of the plasma cooling. 
Fig. (5) Shows the variation in the electron number density with the distance .The number density decreases along the direction of expansion of the plume. The electron density decreases rapidly within a short distance from the sample because of the recombination of plasma particles.

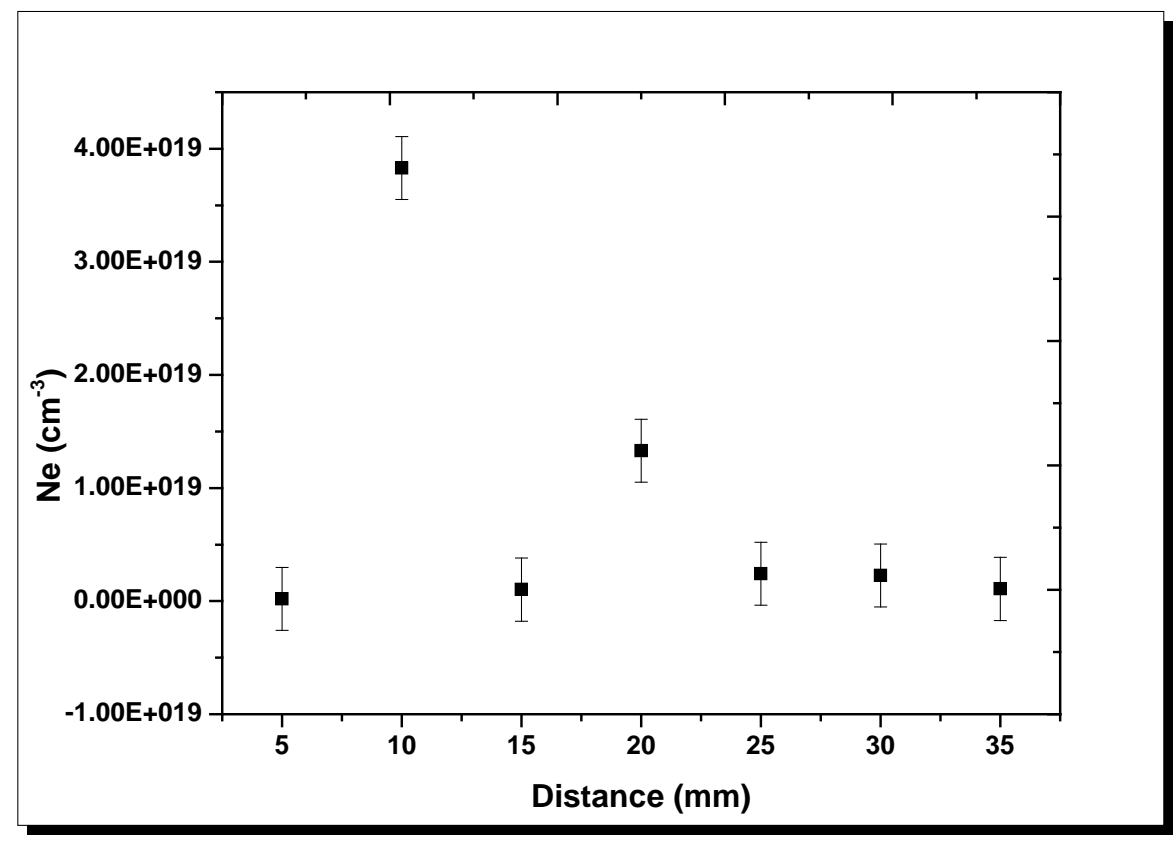

Fig. (5): the variation in the electron number density with the distance at Mo I $465.5 \mathrm{~nm}$ using the fundamental $(1064 \mathrm{~nm})$ wavelength of Nd-YAG laser at fixed power $0.875 \mathrm{~W}$

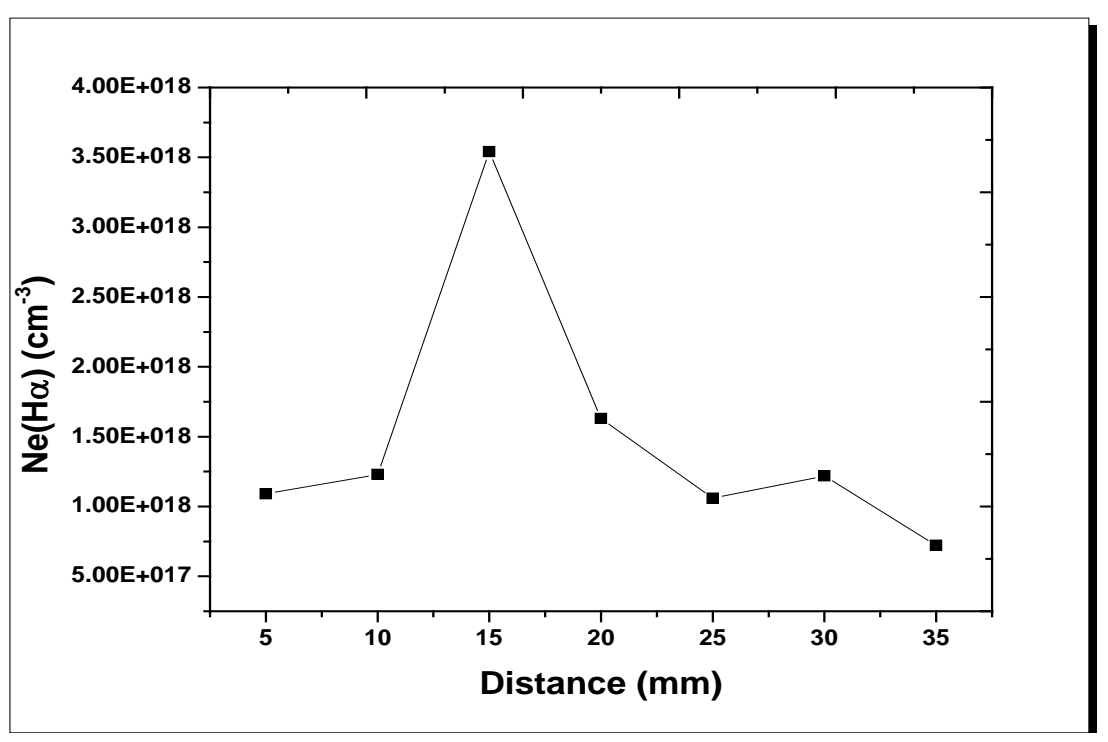

Fig. (6): the variation in the electron number density from $\mathrm{H}_{\alpha}$-line with the distance at Mo I $465.5 \mathrm{~nm}$ Using the fundamental $(1064 \mathrm{~nm})$ wavelength of Nd-YAG laser at fixed power $0.875 \mathrm{~W}$

Similar to Fig (5), Fig (6) shows the use of the electron number density from hydrogen $\mathrm{H} \alpha$-line with the distance using the fundamental (1064 nm) wavelength of the Nd-YAG laser at fixed power $0.875 \mathrm{~W}$. Previous researchers measured the electron density and temperature of hydrogen $\mathrm{H} \alpha-$ line as a function of the delay time [8]. In the present study, the line width and intensity of hydrogen $\mathrm{H}_{\alpha}$-line using eq (2) were utilized to 
determine the electron density and the temperature as a function of distance from the target.

Effect of laser input power

The $\left(\mathrm{N}_{\mathrm{e}}\right.$ and $\left.\mathrm{T}_{\mathrm{e}}\right)$ for different values of laser input power were calculated. We observed that the electron temperature is linearly increased with increasing the laser input power. Figure (7) shows the variation in the electron temperature with the laser input power at a distance of $15 \mathrm{~mm}$ from the target. The laser input power varies from 0.5 to 1.5 $\mathrm{W}$. While the electron temperature varies from $6000 \mathrm{~K}$ to $7500 \mathrm{~K}$.

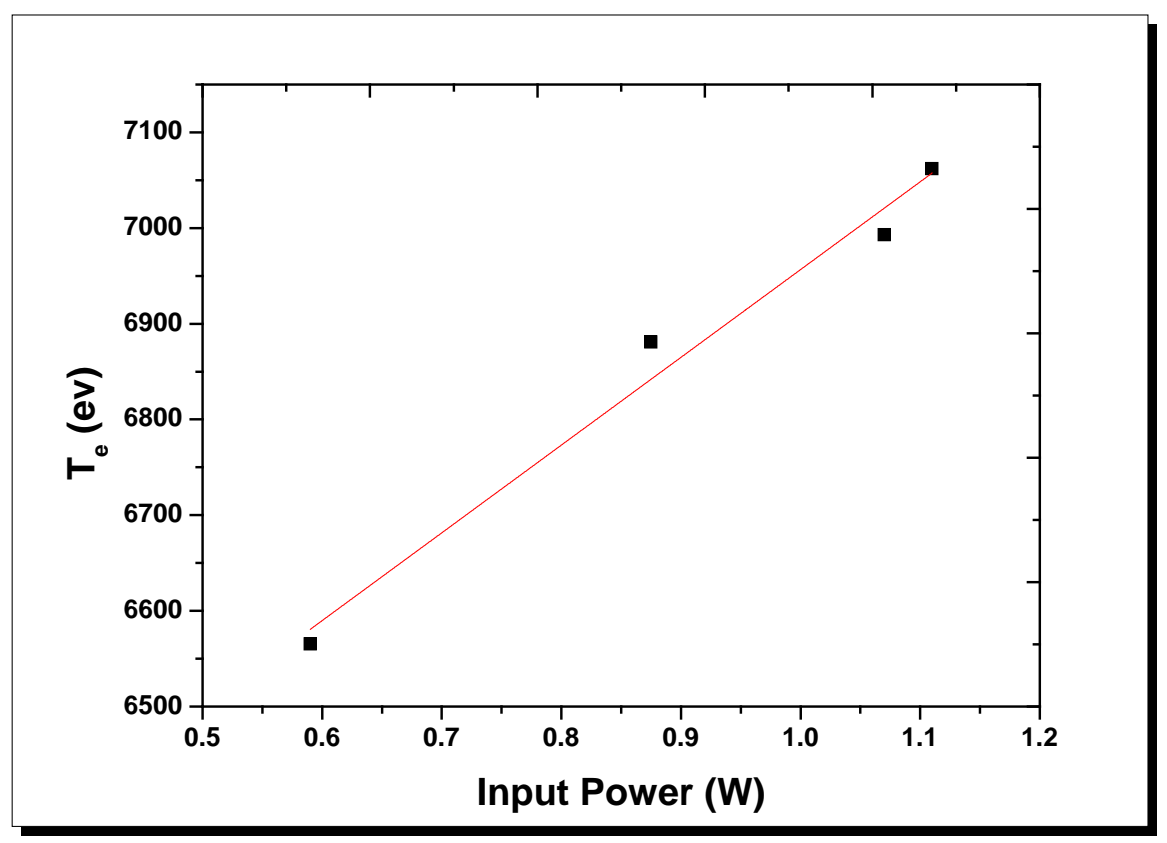

Fig. (7): the variation of electron temperature with input power at fixed distance $15 \mathrm{~mm}$ from the target.

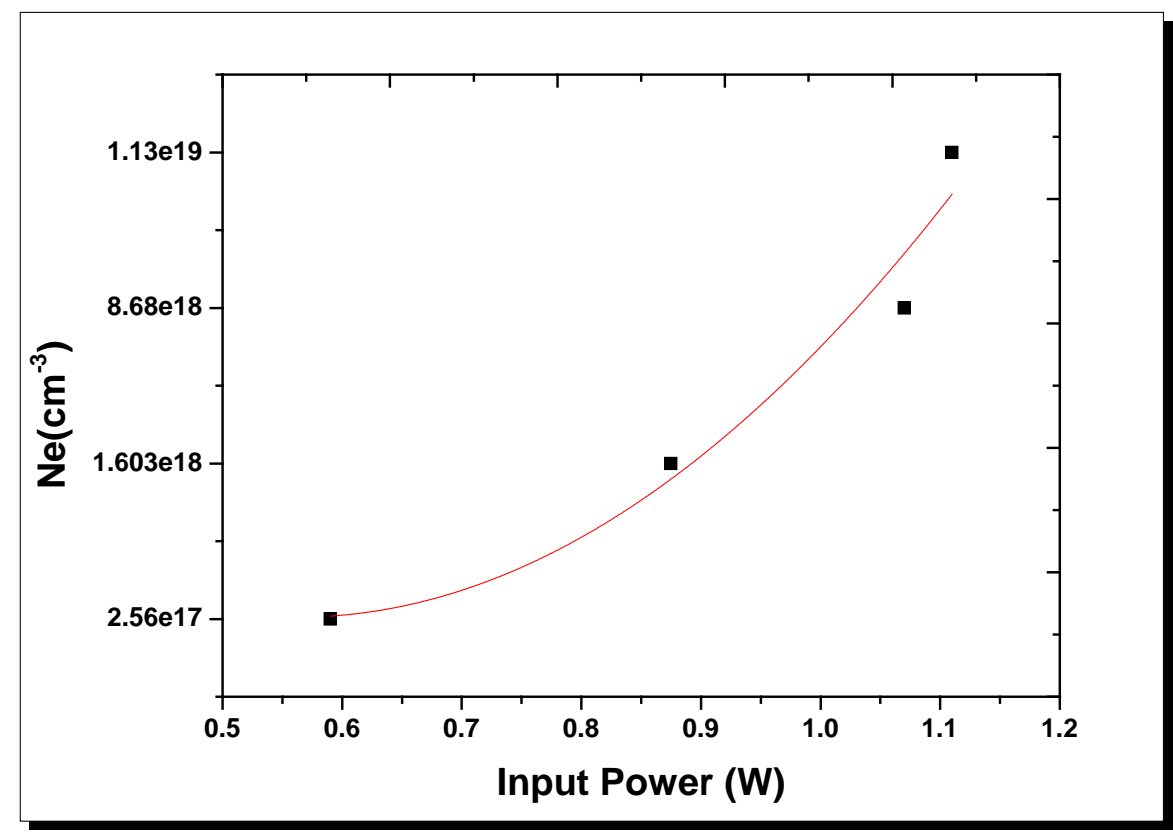

Fig. (8): the variation of the electron density with the input power at $465.5 \mathrm{~nm}$ and fixed distance From the target. 
Fig (8) shows the variation of the electron density as a function of the laser input power .The variation in the electron density with the laser input power has a polynomial increase. Whereas, the electron density changes from $2.56 \times 10^{17}$ up to $1.13 \times 10^{19} \mathrm{~cm}^{-3}$, at a higher laser input power there is an increase in the plasma temperatures and the electron densities.

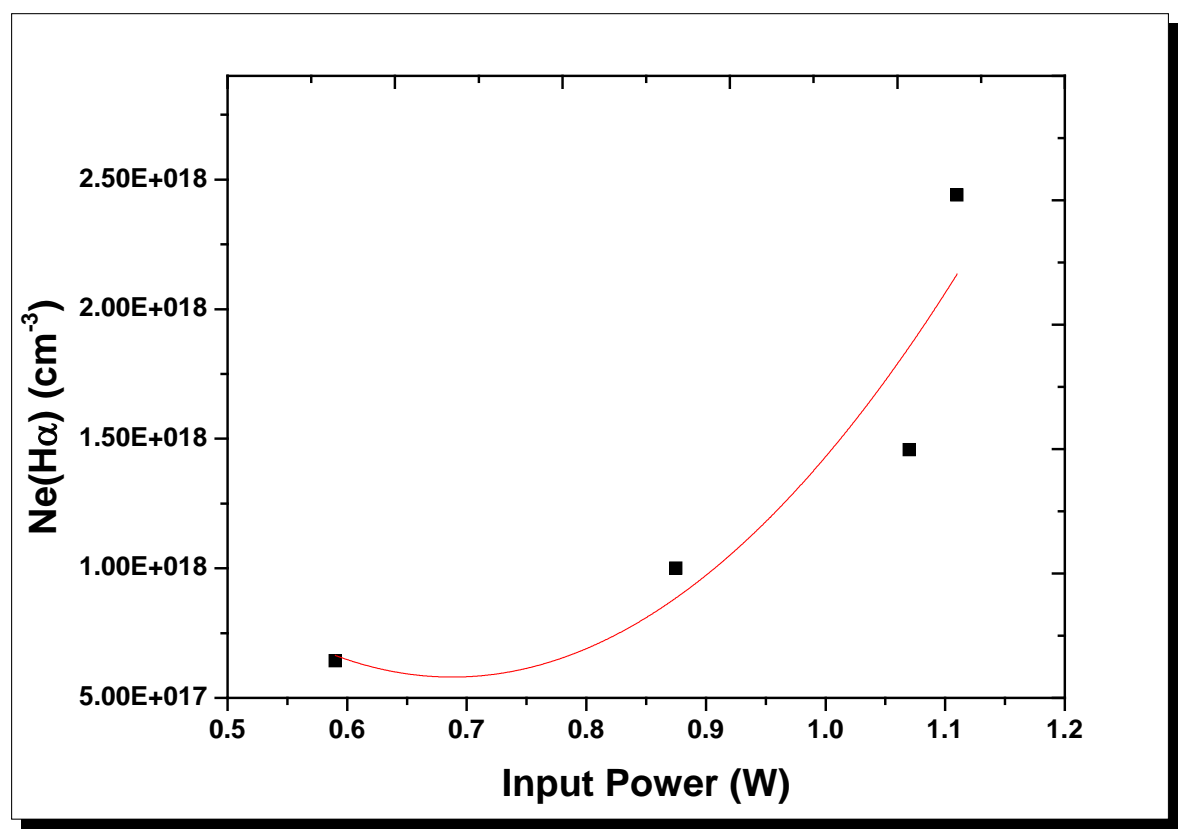

Fig .(9): the variation of electron density from (hydrogen $\mathrm{H}_{\alpha^{-}}$-line) with input power at fixed distance $15 \mathrm{~mm}$

Fig. (9) shows the variation of electron density using the hydrogen $\mathrm{H}_{\alpha}$-line, as a function of the laser input power. The variation in the electron density with the laser input power increases, whereas the electron density ranges from $5 \times 10^{17}$ up to $2.5 \times 10^{19} \mathrm{~cm}^{-3}$. The electron density measured using the $\mathrm{H}_{\alpha}$-line provides more accurate values than those in the literature [8].

\section{Conclusion}

The electron density and electron temperature were calculated using three neutral lines emitted from molybdenum. The plasma parameters were determined via the interaction of Nd-YAG laser beam with a molybdenum solid sample in open air . The parameters of plasma were obtained from Mo lines at 465.5, 498 and $517 \mathrm{~nm}$ as well as those obtained from $\mathrm{H \alpha}$-line at $656.3 \mathrm{~nm}$. The temperature was measured utilizing Boltzman plot. The electron density was calculated from Stark broadening. The studies of the plasma parameters with different input powers and distances of plasma plume showed a change in the electron density and temperature values with the changing of the laser power and the distance of the target.At a distance of $10 \mathrm{~mm}$ from the target , the electron temperature has a minimum value while the electron density shows a maximum value.

\section{References}

1- Mohmoud El Sherbini A, Aziz Saad Al Aamer A. J. Signal and Inform Proces. 03,502-15,(2012).

2- Hanif.M, Salik.M . J.Russ. Las. Research, 35. 3 (2014).

3- Haider Z, Munajat Y, Kamarulzaman R. J. Teknologi.57,(2013).

4- Gaudiuso R, Dell'Aglio M, Pascale OD, Senesi GS, Giacomo AD. Sensors.10,7434-68,(2010).

5- Anabitarte F, Cobo A, Lopez-Higuera JM. ISRN Spectroscopy.2012,1-12,(2012).

6- Sherbini AME, Aamer AASA, Hassan AT, Sherbini TME. Opti and Photo. J. 02,27885(2012).

7- Hanif M, Salik M. Optics and Spectroscopy.116,315-23,(2014). 
8- Sherbini AME, Aamer AASA. World .J.Nano Sci and Engine.02,206-12,(2012).

9- Sherbini AME, Aboulfotouh A-N, Rashid F, Allam SH, Al-Kaoud AM, Dakrouri AE. Natural Sci.05,501-7,(2013).

10- Sherbini AME, Aboulfotouh A-NM, Rashid FF, Allam SH, Dakrouri AE, Sherbini TME. World. J.Nano Sci and Engine.02,181-8,(2012).

11- El Sherbini AM, El Sherbini TM, Hegazy H, Cristoforetti G, Legnaioli S, Palleschi V. Spectrochimica Acta Part B: Atomic Spectro.60,1573-9,(2005).

12- Rashid FF, Elsherbini AM, Al-Muhamady A. Applied Physics A.115,1395-9,(2014).

13- Idris N, Lahna K, Fadhli, Ramli M. J.Physics: Conf Series.846,012020(2017).

14- Jakubowska K, Kubkowska M, Blagoev A, Rosiński M, Parys P, Gąsior P. Physica Scripta.T161,014029,(2014).

15- Lifeng Qi, Lanxiang Sun, Zhibo Cong, Yong Xin, Yang Li. J.Compu and Communic.01,369,(2013).
16- Muhammad asif Naeem YJ, Munawar Iqbal.Asian.J.Chemistry,(2012).

17- Sternberg EMA, Rodrigues NAS, Amorim J. Applied Physics .B,122,(2016).

18- Sherbini AME, Amer AASA, Hassan AT, Sherbini TME. Opti and Photo. J. 02,28693,(2012).

19- Musadiq M, Iqbal M, Amin N, Jamil Y, Naeem MA, Shahzad HA. Intern.J. Engine \& Tech.2,(2012).

20- Rehan I, Muhammad R, Rehan K, Karim K, Sultana S. J.Nutrition \& Food Sciences.07,(2017).

21- Shaikh NM, Rashid B, Hafeez S, Jamil Y, Baig MA. J.Physics D: Applied Physics.39,138491,(2006).

22- Rehan I, Rehan K, Sultana S, Oun ul Haq M, Niazi MZK, Muhammad R. The European Physical .J.Applied Physics.73,10701,(2016).

23- Sternberg EMA, Rodrigues NAS, Amorim J, Sbampato ME. J.Physics: Conf Series.370,012052,(2012). 